\title{
Polyarteritis Nodosa - Risk of Stopping Immunosuppressent and a Rare Fatal Complication
}

\author{
Akhil PAUL'ㄱ, Richa GUPTA', Devasahayam Jesudas CHRISTOPHER ${ }^{1}$
}

\begin{abstract}
Background: Polyarteritis nodosa (PAN) is a systemic necrotising vasculitis which can cause multifocal aneurysms of visceral arteries, which may rupture causing haemorrhage, thrombosis, organ ischemia and infarction While the treatment of vasculitis requires long term use of immunosuppressive drugs, these patients are more prone for diseases like tuberculosis due to decreased cell mediated immunity. Case presentation: Hepatic involvement in PAN is not common and many of the findings are occult. A 40 year old lady, a known case of polyarteritis nodosa on treatment with steroid and immuno-suppressants for the last 18 years, with history of multiple episodes of relapse of the vasculitis, and cyclophosphamide induced ovarian failure on hormone replacement therapy was diagnosed to be having sputum positive tuberculosis. She was initiated on anti-tuberculosis treatment. Vasculitis was on remission for the last 12 months. So Mycophenolate Mofetil was stopped as per the advice of the Rheumatologist. 14 days later she developed abdominal pain with dropping hemoglobin. CT angiography showed ruptured liver hematoma with hemoperitoneum and she succumbed to her illness. Conclusion: Spontaneous hepatic hemorrhage, probably due to a ruptured hepatic artery aneurysm is a rare complication which is reported, only a very few times in the literature so far. This case report throws light on the possible detrimental outcomes in stopping immunosuppressive drugs, especially in a patient with multiple episodes of relapse in the past, in the background of an ongoing infection like tuberculosis. Continuation of the immunosuppressive drugs need to be considered against it's stoppage in high risk patients.
\end{abstract}

Keywords: polyarteritis nodosa, immunosuppressants, complication.

\section{INTRODUCTION}

Polyarteritis nodosa (PAN) is a systemic vasculitis. It is characterized with necrotizing inflammation of the small and medium sized vessels, resulting in microaneurysms, aneurysm rupture and hemorrhage, thrombosis, organ ischemia and infarction ${ }^{1}$. In this case report, we are presenting a lady, who was a diagnosed case of PAN on treatment, who developed liver hematoma, which ruptured to cause hemoperitoneum and later turned to be fatal to the patient.

\section{CASE REPORT}

A 40 year old lady, a known case of Polyarteritis Nodosa on treatment with steroid and immunosuppressent drugs for the last 18 years, steroid induced diabetes mellitus, hypothyroidism and Cyclophosphamide in-
1 Department of Pulmonary Medicine, Christian Medical College Hospital, Tamil Nadu, India

\section{Corresponding author.}

Akhil PAUL, Christian Medical College Hospital, Vellore - 632004, Tamil Nadu, India.

E-mail: akhil_pauli@yahoo.com 
duced premature ovarian failure presented to us with low grade intermittent fever, cough with minimal mucoid expectoration and loss of appetite for 3 months. There was no history of breathlessness, chestpain, hemoptysis or altered bowel or bladder habits. She was diagnosed to be having have sputum positive tuberculosis at her homeland, with Acid Fast Bacilli smear $2+$, one month back. Since then, she was on daily regimen of Category-I anti-tuberulosis drugs. In the past, she had multiple episodes of relapse of the vasculitis with skin nodules and hematuria. However, for last 12 months she was in disease remission. Currently she was on Mycophenolate Mofetil. For the last 12 months, she was on disease remission and tapering dose of steroids.

Her General examination revealed that, she was under weight with a BMI of $16.9 \mathrm{~kg} / \mathrm{m}^{2}$. She was pale, tachycardic and tachypnoiec. She was tachycardic and tachypnoeic at presentation. Jugular venous pressure was not elevated and she was pale. Respiratory system examination revealed bronchial breath sound in the right infra-scapular region. Other systemic examination was unremarkable. There was bronchial breathsound heard in the right infra-scapular region. Her chest $\mathrm{x}$ ray (Figure 1) showed right lower lobe consolidation. Her WBC count was $14,500 \mathrm{cell} / \mathrm{mm}^{3}$ and hemoglobin was $10.5 \mathrm{~g} \%$. As the sputum evaluation including Mycobacterial culture and TB-PCR failed to demonstrate the presence of mycobacterium tuberculosis and Computed Tomography of Thorax showed cavity- consolidation of the right lower lobe, she underwent bronchoscopy and trans bronchial lung biopsy (TBLB). She developed post TBLB pneumothorax which was managed using an inter costal draining tube, which was

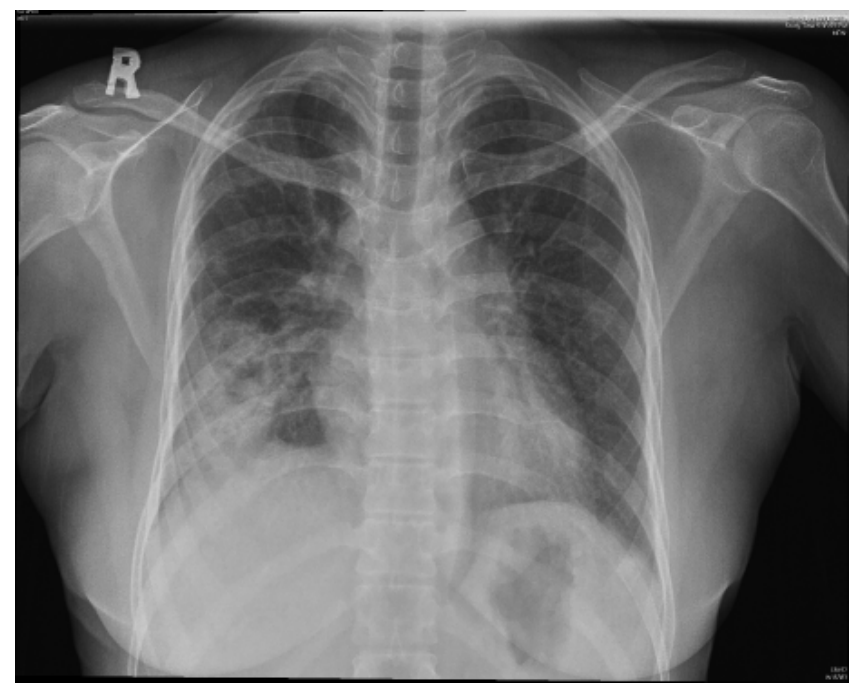

Figure 1. Chest radiography showing right lower lobe consolidation. later removed. The bronchial lavage sample and lung biopsy tissue sample demonstrated mycobacterium tuberculosis with no rifampicin resistance in the TB$\mathrm{PCR}$ test and later grew drug sensitive mycobacterium tuberculosis in the culture. Histopathology of the lung tissue showed necrotizing granulamatous lesion. So it was decided to continue on the anti-tuberculosis therapy. Mycophenolite mofetil was stopped as per the advice of the Rheumatologist, in the background of ongoing tuberculosis. She was also started on IV Piperazillin and Tazobactem according to the sensitivity showed by Klebsiella, which grew in the bronchial lavage culture. 2 weeks later she started complaining of abdominal pain.

There was drop in hemoglobin by $1.5 \mathrm{~g} \%$ from the baseline. There was no worsening of the lung opacity in the repeated chest X-ray. She continued to have low grade fever. There was mild increase in the total WBC counts, but there was no band forms. Evaluation for other infections including blood cultures were negative and her serum procalcitonin level was only 1.2 $\mathrm{ng} / \mathrm{ml}$. Ultrasound abdomen also did not demonstrate any abnormal finding. Her hemoglobin level dropped further drastically by $3 \mathrm{~g} \%$, which required blood transfusions of 2 units packed cells. As she continued to complain of diffused abdominal pain, her blood lactate levels and liver enzymes started rising. with rising blood lactate levels and liver enzymes ,bowel ischemia secondary to worsening of vasculitis was suspected and she underwent $\mathrm{CT}$ angiography of the abdomen with reconstruction study (Figure 2). It showed a large $9 \mathrm{x}$ $12 \times 15 \mathrm{~cm}$ partially organized hematoma in the right

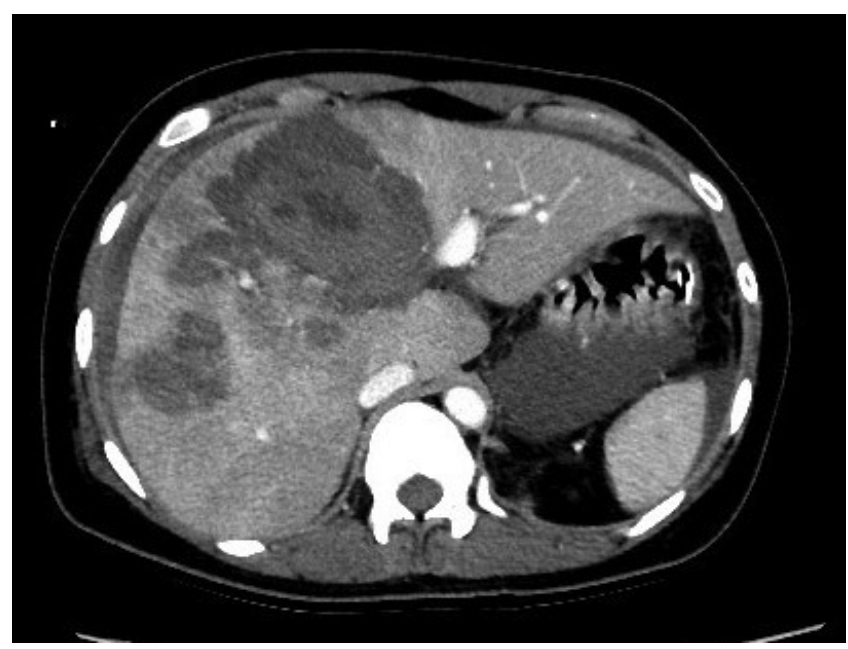

Figure 2. Computed tomographic angiography of the abdomen showing ruptured aneurysm in the liver causing parenchymal hemorrhage. 
lobe of liver, active bleed in the hepatic parenchyma and hemoperitoneum. A family meeting was held with multi-department representation including, Pulmonologists, Rheumatologist and the Hepato-biliary surgeon and the condition of the patient and poor prognosis was explained. According to the decision taken by the relatives, she was conservatively managed in the ward with blood transfusions and other medications, and later she succumbed to her illness after 2 days.

\section{DISCUSSION}

Polyarteritis nodosa affects multiple systems including joints, skin, gastro-intestinal tract and kidney. Hepatic involvement in PAN is not common and many of the findings are occult. In 1977, Cowan et al reported 2 cases with hepatic arteriole involvement ${ }^{1}$. In that, histopathology of the hepatic tissue demonstrated fibrinoid necrosis of the hepatic arteriole media in one patient and centrilobular congestion and necrosis with periportal fibrous tissue calcification in another. In a radiographic study done by Stanson et al. ${ }^{2}$, they did angiographic examination of the abdominal vessels of the patients diagnosed to be having PAN. Out of 36 patients, evaluated for hepatic vessel abnormality, 32 patients (89\%) had various involvement of the vessels including occlusion, aneurysm and ectasia. Aneurysms can rupture to cause serious hemorrhage and thrombosis can lead to infarction of the organ. Our patient had a spontaneous hepatic hemorrhage to form a large hematoma, which later ruptured to cause hemoperitoneum. The initial hemorrhage could have been secondary to a ruptured hepatic artery aneurysm, as a result of the relapse of PAN on stopping immunosuppressive medication. There are only, a few case reports on spontaneous liver rupture, in the literature so far. Even

\section{References}

1. Cowan RE, Mallinson CN, Thomas GE, Thomson AD. Polyarteritis nodosa of the liver: a report of two cases. Postgrad Med J. 1977 Feb;53(616):89-93.

2. Stanson AW, Friese JL, Johnson CM, McKusick MA, Breen JF, Sabater EA, et al. Polyarteritis Nodosa: Spectrum of Angiographic Findings. RadioGraphics. 2001 Jan 1;21(1):151-9.

3. Li AKC, Rhodes JM, Valentine AR. Spontaneous liver rupture in polyarteritis nodosa. Br J Surg. 1979 Apr 1;66(4):251-2.

4 Battula N, Tsapralis D, Morgan M, Mirza D. Spontaneous liver haemorrhage and haemobilia as initial presentation of undiagnosed polyarteritis nodosa. Ann R Coll Surg Engl. 2012 May;94(4):e163-5. though Ayers and Fitchett had reported a case of liver parenchymal bleed in a case of PAN, Li et al. in 1979 had reported the first case of spontaneous liver rupture 3 , as that happened in our patient. Later Batulla $\mathrm{N}$ et al in $2012^{4}$ and Irene Gomez- Luque et al. in $2016^{5}$, reported cases of spontaneous liver haemorrhage or rupture as initial presentation of undiagnosed Polyarteritis nodosa.

Also, patients on immuno-suppressants are more prone for diseases like tuberculosis due to decreased cell mediated immunity, especially those who are on Prednisolone at a dose $>15 \mathrm{mg} /$ day or its equivalent or anti TNF agents 6 . There is increased incidence of tuberculosis with other drugs including mycophenolite mofetil as well ${ }^{7}$. Even though there is no specific guidelines on stopping or decreasing immunosuppression on development of tuberculosis, it is advisable to bring down the immunosuppressant dose to the possible minimum.

\section{CONCLUSION}

We are reporting a case of spontaneous liver rupture in a case of polyarteritis nodosa, which is a very rare fatal complication. And this case report also throws light to the possible detrimental outcomes in stopping immunosuppressive drugs, especially in a patient with multiple episodes of relapse in the past, in the background of an ongoing infection like tuberculosis.

Compliance with ethics requirements: The authors declare no conflict of interest regarding this article. The authors declare that all the procedures and experiments of this study respect the ethical standards in the Helsinki Declaration of 1975, as revised in 2008(5), as well as the national law. Informed consent was obtained from all the patients included in the study.

5. Irene Gómez-Luque, Felipe Alconchel, Rubén Ciria, M Dolores Ayllón, Antonio Luque, Marina Sánchez et al, Spontaneous liver rupture as first sign of polyarteritis nodosa. World $\mathrm{J}$ Hepatol. 2016 Nov 18; 8(32): 1414-1418.

6. Horsburgh CR, Rubin EJ. Clinical practice. Latent tuberculosis infection in the United States. N Engl J Med. 2011 Apr 14;364(15):1441-8.

7. Mercadal L, Foltz V, Isnard-Bagnis C, Ourahma S, Deray G. Tuberculosis after conversion from azathioprine to mycophenolate mofetil in a long-term renal transplant recipient. Transplant Proc. 2005 Dec;37(10):4241-3. 
